\title{
Characterization of Plasma Treated Surfaces for Food Safety by Terahertz Spectroscopy
}

\author{
Kateřina Sulovskáa ${ }^{a, b^{*}}$, Marián Lehockýb \\ ${ }^{\mathrm{a}}$ Faculty of Applied Informatics, Department of Security Engineering, ${ }^{\mathrm{b}}$ University Institute, Centre \\ of Polymer Systems
}

Tomas Bata University in Zlín, Nam. T. G. Masaryka 5555, 76001 Zlín, Czech Republic

\begin{abstract}
:
A physico-chemical approach to modify surfaces not only for use in medicine, but also for preservation of food is nowadays widely studied to lower the risks of increased number of bacterial pathogens that are in a direct contact with people. Food safety is very important part of preserving sustainability during crises, especially after the enterohaemorrhagic Escherichia coli outbreak in Europe in 2011. One of the possibility how we can protect food against various pathogens is the modification of packing materials that are directly in contact with preserved food. This contribution deals with the characterization of modified surfaces with antibacterial properties via Terahertz spectroscopy. For the purpose of this paper, three monomers were used for grafting onto air radiofrequency plasma activated low density polyethylene surface, which created a brush-like structure. Next, the antibacterial agents, Irgasan and Chlorhexidine, were anchored to these surfaces. These antibacterial agents were selected for supposed effect on two most frequently occurring bacterial strains - Escherichia coli and Staphylococcus aureus. Materials were further tested for the presence of antibacterial agent molecules, in our case by means of terahertz spectroscopy. Each material was tested on two spectroscopes - the SPECTRA and the OSCAT terahertz instruments.
\end{abstract}

Keywords: antibacterial surface, terahertz time-domain spectroscopy, pulsed terahertz ATR spectroscopy, refractive index, absorbance.

\section{Introduction}

Outbreaks of Escherichia Coli (E. Coli) and other pathogens are known for centuries and returns repeatedly in various periods and forms. The hazard of infections mostly comes from contaminated food, water (beverages), animals or even people. As far as the animals and people can be infected and treated in many ways, the simplest way to prevent taints as those in Europe (2011, 2001, 1920), Japan (1996), USA (1993, 1976), etc. is to preclude contamination of what is consumed. One of the easiest ways how to do it is to use modified packages. Active packaging systems have been successfully applied mainly in USA, Japan and Australia. Their significant development in Europe was broken due to more strict regulations on packaging materials used for contact with food, and absence of regulations for practical use. The main function of packaging materials is to protect a product from degradation. The other functions are also indispensable - they enable easy manipulation and visual communication with customers, extend durability, heighten

*sulovska@fai.utb.cz 
safety and intensify sensory properties (active packages), or monitor conditions of packed products during transportation and stocking (intelligent packages). ${ }^{1}$ Active packages are solution in cases when the number of health-endangering pathogens needed to be decreased to protect the product from degradation and human health from infections. In most solid/semi-solid foods the microbial growth is apparent mainly on their surface, in processed and mixed food the growth can be notices thorough the product. Antibacterial packages can be also used in cases of thermally sterilized food or food with self immune system, because these products may be also contaminated (e.g. by defects in package, contamination after opening). The major task of antibacterial packaging materials in food industry is to reduce the speed of growth of microorganisms to prolong the shelf life and to ensure the food safety. Thus, the risk of outbreaks of dangerous microorganisms can be lowered by the help of modern active packaging materials at the source.

Such antibacterial surface for packaging purposes must be also characterised after manufacturing to know whether the antibacterial surfactant is attached to the polymer or not. One of the fast developing methods in the last years for characterizing various materials is terahertz (time-domain, pulsed) spectroscopy. Terahertz spectroscopy is also known for its utilization in security industry (e.g. airport scanners, detectors of drugs or explosives), but the terahertz radiation can be also used for characterization of materials and their imaging (e.g. showing layers where possible or cracks in material). Terahertz spectroscopy is also very promising technology for studying and understanding intermolecular (vibrational) frequencies and forces of the complexes ${ }^{2}$.

The aim of this paper is to demonstrate the possibility of detecting specific vibrations of antibacterial agents bonded by intermolecular forces on monomers for functionalization grafted onto air plasma activated surfaces by the terahertz spectroscopy. The absorbance spectra are assigned where possible.

\section{Materials \& Methods}

\subsection{Samples}

The antibacterial surfaces were prepared according to the procedure used in previous research ${ }^{3}$. This time, two different antibacterial surfactants, triclosan and chlorhexidine, were anchored onto air plasma treated low density polyethylene (LDPE) grafted with three monomers, allylamine (AA), $N$-allylmethylamine (AMA), $N, N$-dimethylallylamine (DMAA). LDPE was chosen as it is the most commonly used packaging material in the food industry. The low-temperature plasma treatment was selected for reasons of extent of modifications which can be easily controlled by treatment conditions ${ }^{4-6}$ without losing desirable mechanical characteristics of bulk polymer.

\subsection{Instrumentation}

The main work was done on the TPS ${ }^{\mathrm{TM}}$ Spectra 3000 by TeraView Ltd., which can measure in several modes depending on modules connected to the main body of the spectrometer. The presented results were achieved using an ATR module, which measures spectra by direct contact of sample with the module inserted on the workplace of the device. The measured data of prepared samples are processed via device software

The spectrometer was operating with resolution of $1.2 \mathrm{~cm}^{-1}$ ( $32 \mathrm{GHz}$ ), scanning frequency of 30 scans per second, and each measurement contained 100 scans in each point.

Another set of collected spectra (refractive indexes) came from the Tera OSCAT by Menlo Systems GmbH, which works as a classical terahertz time-domain spectroscopy (THz-TDS) system based on the pump-probe principle, which is based on a direct control of the time delay between the pulses by varying the pulse repetition rate of the laser source without any external mechanical delay line or two synchronized femtosecond lasers. ${ }^{7,8}$ The experimental setting had a standard transmission geometry (see Fig. 1). The laser source is a mode-locked femtosecond Er-doped fibre oscillator with $90 \mathrm{fs}$ pulses at $1560 \mathrm{~nm}$ at a repetition rate of $250 \mathrm{MHz}$. 

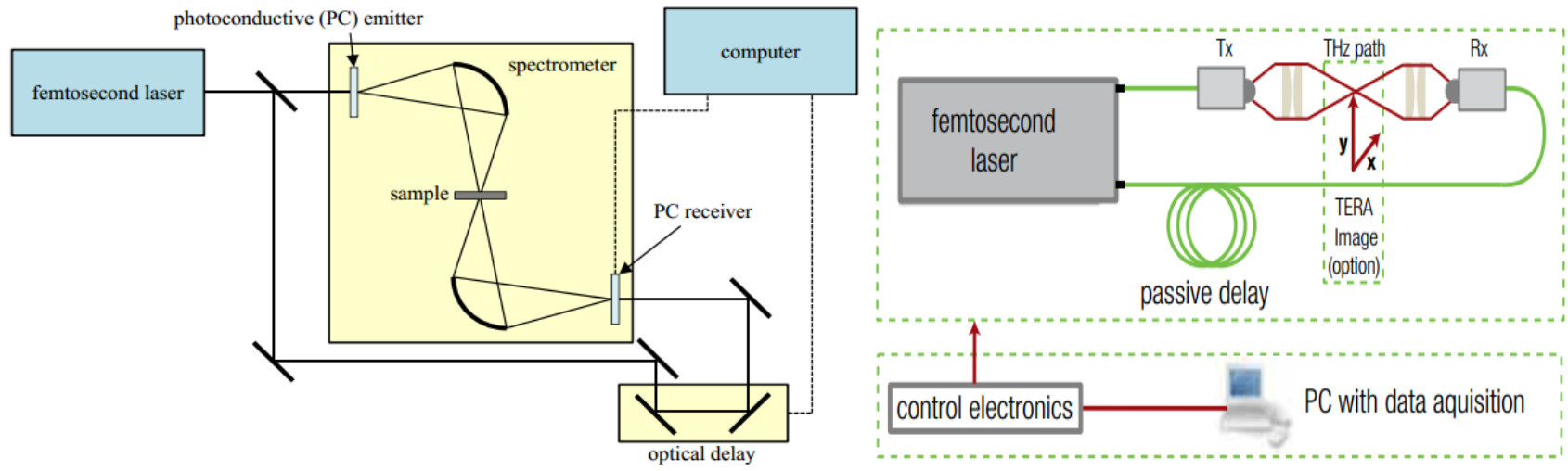

Figure 1. Schematic TPI Spectra 3000 showing coherent detection mechanism (left) $)^{9}$, Tera OSCAT spectrometer layout (right) ${ }^{8}$

The reference was measured for both instruments only through air. Each sample was measured three times in case of SPECTRA and ten times in case of OSCAT in several places to control the accuracy of experiment. Thickness of each sample was measured at ten different places and averaged to get representative values of material for a total overview of selected behaviour in $\mathrm{THz}$ frequency. The measurements were not done under special conditions (e.g. nitrogen atmosphere), so the spectral contributions associated with atmospheric moisture may occurred, which in fact is not a problem for the experiment itself as we were looking for special vibrations of antibacterial surfactants. The frequency range of the experiment using the Tera OSCAT instrument was set from $0 \mathrm{THz}$ to $2.6 \mathrm{THz}$, and $0 \mathrm{THz}$ to $4 \mathrm{THz}$ using TPS Spectra ATR module.

\section{Results and Discussion}

Observed signals from THz-TDS and ATR THz were, as well as the previously collected spectra from ATR FTIR, low intensity. However, changes in treated materials are visible as can be seen from obtained transmittance and refractive indexes and can differentiate each material. The absorbance (absorbance coefficient) from the Tera OSCAT are not subject to this paper due to their very low values caused by the thickness of materials. All data were evaluated using the Statistica 7 by StatSoft Ltd.

Table 1. Descriptive statistics of refractive indices (by Tera OSCAT)

\begin{tabular}{lcccccc|cc}
\hline \multicolumn{1}{c}{ Sample (No.) } & Mean & $\begin{array}{c}\text { Standard } \\
\text { deviation }\end{array}$ & Median & Min & Max & $\begin{array}{c}\text { Standard } \\
\text { error }\end{array}$ & $\begin{array}{c}\text { Thickness } \\
{[\boldsymbol{\mu m}]}\end{array}$ & $\begin{array}{c}\text { Standard } \\
\text { deviation }\end{array}$ \\
\hline PE (1) & 2.1008 & 0.0004 & 2.1280 & -1.6330 & 4.0550 & 0.0037 & 95.3 & 0.0013 \\
PE plasma (2) & 3.2776 & 0.0007 & 3.3040 & -3.3570 & 8.0520 & 0.0019 & 83.7 & 0.0014 \\
PE plasma AA (3) & 2.6441 & 0.0008 & 2.4690 & -2.3790 & 5.2850 & 0.0056 & 87.8 & 0.0007 \\
$\begin{array}{l}\text { PE plasma AA } \\
\text { Chlorhexidine (4) }\end{array}$ & 3.2259 & 0.0007 & 3.2300 & -3.2360 & 7.8100 & 0.0049 & 84.6 & 0.0010 \\
$\begin{array}{l}\text { PE plasma AA } \\
\text { Triclosan (5) }\end{array}$ & 3.0614 & 0.0007 & 2.9890 & -2.6750 & 7.0240 & 0.0039 & 96.2 & 0.0073 \\
$\begin{array}{l}\text { PE plasma DMAA (6) } \\
\text { PE plasma DMAA }\end{array}$ & 2.8732 & 0.0006 & 2.8880 & -2.6360 & 6.7850 & 0.0030 & 99.5 & 0.0018 \\
$\begin{array}{l}\text { Chlorhexidine (7) } \\
\text { PE plasma DMAA }\end{array}$ & 2.9723 & 0.0006 & 3.0020 & -2.7860 & 6.9880 & 0.0037 & 95.7 & 0.0009 \\
$\begin{array}{l}\text { Triclosan (8) } \\
\text { PE plasma AMA (9) }\end{array}$ & 3.1888 & 0.0007 & 3.2170 & -3.2990 & 7.7050 & 0.0039 & 87.3 & 0.0007 \\
$\begin{array}{l}\text { PE plasma AMA } \\
\text { Chlorhexidine (10) }\end{array}$ & 3.2958 & 0.0008 & 3.0370 & -2.6660 & 7.1720 & 0.0011 & 99.6 & 0.0038 \\
$\begin{array}{l}\text { PE plasma AMA } \\
\text { Triclosan (11) }\end{array}$ & 3.4247 & 0.0001 & 3.1190 & -3.2190 & 7.5130 & 0.0049 & 84.3 & 0.0040 \\
\hline
\end{tabular}


Each type of surface has its typical range of refractive index values (see Table 1). In contrast to pristine LDPE, all tested samples show changes in their surfaces, not only in displayed peaks but also in shift of the whole behaviours (Fig. 2). Differences in behaviours can be seen from the first step of a physico-chemical preparation of substrates. Each of the samples (No. 1, 2, 3, 6,9) has shifted refractive index behaviour of approx. 0.2 from the previous one. The biggest peaks occur at $0.93 \mathrm{THz}$ for each sample and the highest one belongs to plasma treated LDPE sample. Around $0.55 \mathrm{THz}$ and $1.39 \mathrm{THz}$ a slight decrease of peaks (No. 2,9) can be noticed after magnification. Small peaks around $1.15 \mathrm{THz}$ can be considered as typical for plasma treated LDPE surfaces as well as behaviour between $0.10-0.16 \mathrm{THz}$ region as they occur in all samples except clear LDPE. Other peaks vary mainly within materials with antibacterial surfactant that are bonded by intermolecular forces. The region between $1.65-1.74 \mathrm{THz}$ shows changes in refraction due to plasma treatment and consequent bonding of monomers; while most behaviour proceed similarly, samples No. 3, 5, 9, 10 have attenuated regions, practically to the levels at $1.50 \mathrm{THz}$. Another case of varied (subdued) behaviour may be seen at around $1.95 \mathrm{THz}$ noticeably for the surfaces No. 3, 5, 6, 7, 8. The most significant changes in refractive indices begin beyond the $2 \mathrm{THz}$, where in almost all cases dramatically raises the difference between all modified materials, which helps to distinguish them from each other, except the case of two groups of samples (No. $2 \& 4 \& 8$, and 6 \& 7) that are extremely resembling, which can be also seen in Tab. 1. The greatest shift in behaviour beyond $2 \mathrm{THz}$ border can be observed in all samples with incorporated AMA monomer.

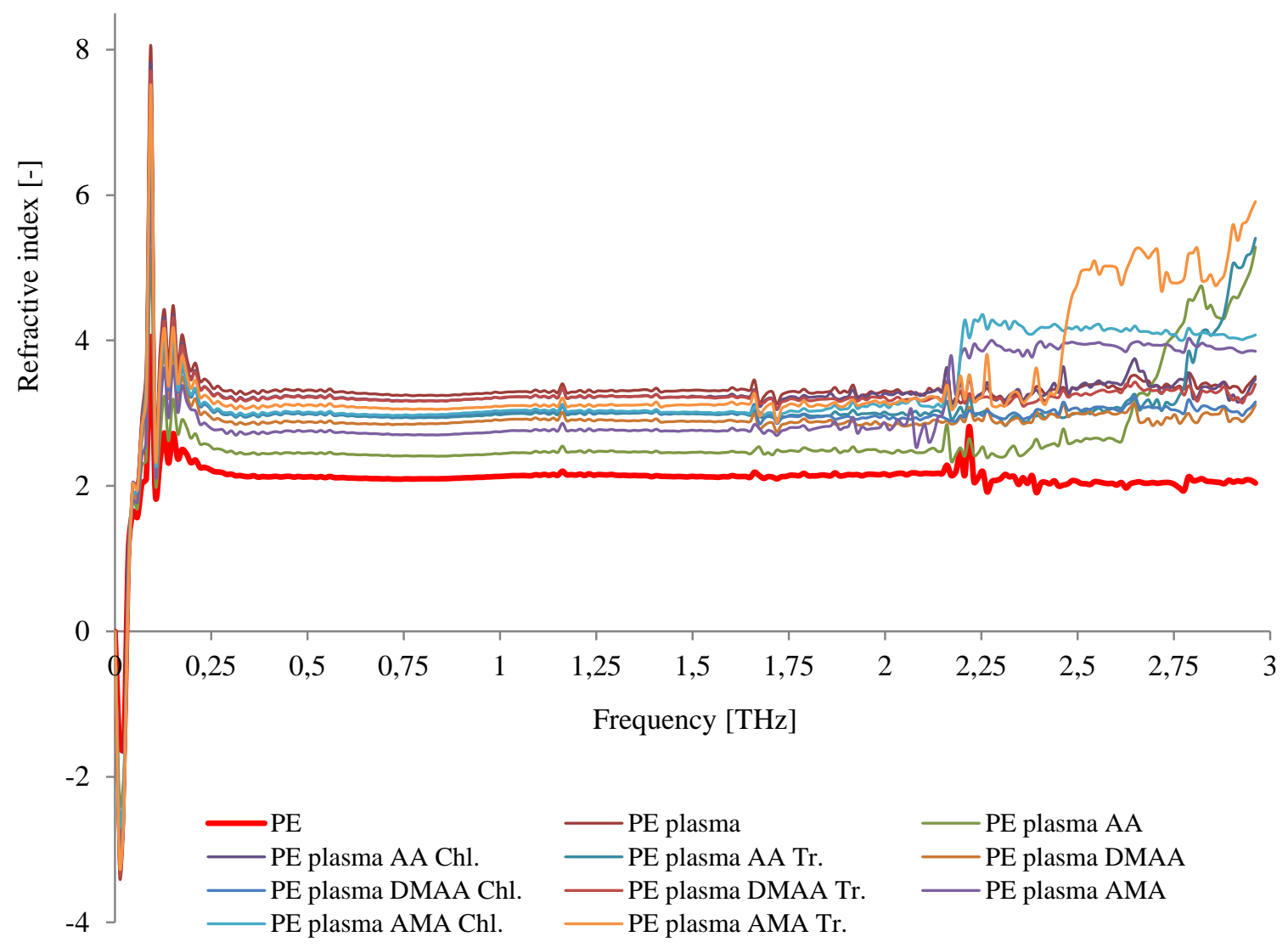

Figure 2. Refractive indices of measured surfaces by Tera OSCAT

In comparison to refractive indices obtained by ATR module (Tab. 2), the distinction of each modified material is easier by the classical transmission, as the ATR refractive indices are due to attenuation very similar and an attempt to distinguish between them may be therefore not accurate. On the other hand, ATR module brings insight into composition of material that may be omit during the utilization of classic transmission chamber (module) or other modules. 
Table 2. Descriptive statistics of refractive indices (experimentally measured by ATR module on TPS Spectra 3000)

\begin{tabular}{lcccccc|cc}
\hline \multicolumn{1}{c}{ Sample (No.) } & Mean & $\begin{array}{c}\text { Standard } \\
\text { deviation }\end{array}$ & Median & Min & Max & $\begin{array}{c}\text { Standard } \\
\text { error }\end{array}$ & $\begin{array}{c}\text { Thickness } \\
{[\boldsymbol{\mu m}]}\end{array}$ & $\begin{array}{c}\text { Standard } \\
\text { deviation }\end{array}$ \\
\hline PE (1) & 1.9471 & 0.0001 & 1.9572 & 0.1055 & 3.3092 & 0.0048 & 95.3 & 0.0013 \\
PE plasma AA (3) & 1.9455 & 0.0001 & 1.9571 & 0.2390 & 2.9253 & 0.0043 & 87.8 & 0.0007 \\
$\begin{array}{l}\text { PE plasma AA } \\
\text { Chlorhexidine (4) }\end{array}$ & 1.9472 & 0.0001 & 1.9573 & 0.0034 & 3.1329 & 0.0047 & 84.6 & 0.0010 \\
$\begin{array}{l}\text { PE plasma AA } \\
\text { Triclosan (5) }\end{array}$ & 1.9381 & 0.0001 & 1.9566 & 0.3680 & 2.1880 & 0.0044 & 96.2 & 0.0073 \\
PE plasma DMAA (6) & 1.9413 & 0.0001 & 1.9578 & 0.0240 & 2.2539 & 0.0042 & 99.5 & 0.0018 \\
$\begin{array}{l}\text { PE plasma DMAA } \\
\text { Chlorhexidine (7) }\end{array}$ & 1.9467 & 0.0001 & 1.9574 & 0.1056 & 3.2236 & 0.0053 & 95.7 & 0.0009 \\
$\begin{array}{l}\text { PE plasma DMAA } \\
\text { Triclosan (8) }\end{array}$ & 1.9487 & 0.0001 & 1.9576 & 0.2529 & 3.2850 & 0.0046 & 87.3 & 0.0007 \\
$\begin{array}{l}\text { PE plasma AMA (9) } \\
\text { PE plasma AMA }\end{array} \quad 1.9463$ & 0.0001 & 1.9568 & 0.2610 & 2.8818 & 0.0037 & 88.4 & 0.0019 \\
$\begin{array}{l}\text { Chlorhexidine (10) } \\
\text { PE plasma AMA }\end{array}$ & 1.9465 & 0.0001 & 1.9570 & 0.3048 & 3.3565 & 0.0045 & 99.6 & 0.0038 \\
Triclosan (11) & 1.949 & 0.0001 & 1.9574 & 0.5050 & 3.0054 & 0.0045 & 84.3 & 0.0040 \\
\hline
\end{tabular}

Characterization of our surfaces via ATR module on TPS Spectra shows changes in spectrum strength between each material. The first changes that can differentiate each spectrum are in the $0-0.2 \mathrm{THz}$ region that is connected with monomers functionalization (biggest peaks for samples No. 8, 10) and can be also a signature of methyl torsion group. A very small peak at $1.7 \mathrm{THz}$ belongs to $\mathrm{N}-\mathrm{H}$ vibration, and the same intensity peak appears at $1.9 \mathrm{THz}$ (C-C vibration). Behaviours in the region of $2-4 \mathrm{THz}$ are very good visible. The area including 2, 2.2, $2.4 \mathrm{THz}$ contains bonds with nitrogen (e.g. primary/secondary amines). The biggest peak in this area is in the position of $2.3 \mathrm{THz}$ (sample No. 8) and belongs to transitions between torsional levels of $\mathrm{CH}_{3}$ group (also at around $2.75 \mathrm{THz}-$ sample No. 4). The peak at 2.9 $\mathrm{THz}$ match with the vibration of nitrogen developed during functionalization of prepared surfaces, same as it is for the $\mathrm{O}-\mathrm{H}$ vibrations at $2.5 \mathrm{THz}$. The space between $3.6 \mathrm{THz}$ and $4 \mathrm{THz}$ is supposed to belong to vibration of bonds with chlorine and other compounds (e.g. $\mathrm{CH}_{2}, \mathrm{CH}, \mathrm{NH}_{2}$, aromatic ring) if possible to detect them in this part of spectrum.

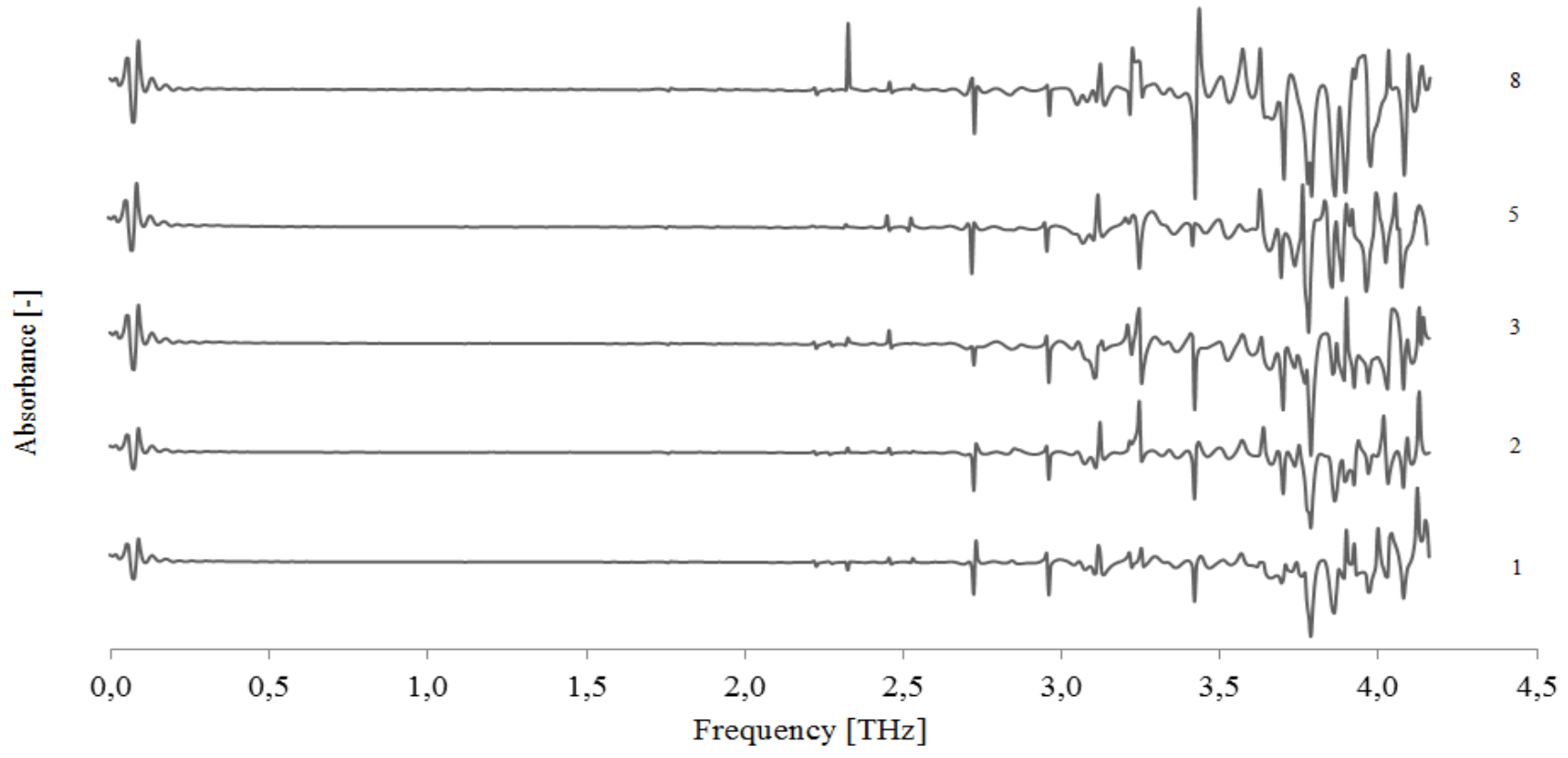

Figure 3. Examples of absorbance spectra measured by ATR module - TPS Spectra 3000 


\section{Conclusion}

Terahertz spectroscopy is mostly used to describe changes in states in biomolecules, respectively biological samples (e.g. oils, tissues, fatty acids) that seem to be more sensitive in the terahertz range that rotational and vibrational state of organic/inorganic molecules. Using the terahertz spectroscopy for characterization of antibacterial surfaces prepared by multistep physico-chemical approach is basically one of the first of its kind. According to our results, it can be said that the terahertz spectroscopy can be also utilized for such characterization if a suitable system is used depending on initial conditions or expected results. However, the issue of insufficiency of described molecules and their peak positions arises during analyses, so that materials and their structures cannot be assigned absolutely.

The further work will consist of measuring dielectric characteristics and other optical properties of antibacterial surfaces by transmission spectroscopic module and reflectance imaging module (to acquire thickness maps and cross sectional images). In the long run, it will be necessary to collect wide range of spectra and characteristic peaks of, at least commonly used molecules.

\section{Acknowledgement}

This work was supported by the IGA grant at Tomas Bata University in Zlín (No. IGA/FAI/2014/001, IGA/FAI/2014/041), by the European Regional Development Fund under the project CEBIA-Tech (CZ.1.05/2.1.00/03.0089), and by the Ministry of Education, Youth and Sport of the Czech Republic under the project Centre of Polymer Systems (CZ.1.05/2.1.00/03.0111).

\section{References}

[1] Kenawy, E.R., Worley, S.D. and Broughton, R., "The chemistry and applications of antimicrobial polymers: A state-of-the-art review“, Biomacromolecul., 8, 1359-1384 (2007)

[2] Tanaka, K., Harada, K. and Yamada, K.M.T., THz and Submillimeter-wave Spectroscopy of Molecular Complexes, [Handbook of High-resolution Spectroscopy], John Wiley \& Sons, Ltd., 853-896 (2011)

[3] Bílek, F., Kř́žová, T. and Lehocký, M., "Preparation of active antibacterial LDPE surface through multistep physicochemical approach: I. Allylamine grafting, attachment of antibacterial agent and antibacterial activity assessment", COLSUB, 88, 440-447 (2011)

[4] Cvelbar, U., "Removal of a thin hydrogenated carbon film by oxygen plasma treatment", Mater. Tehnol., 45, 179$183(2011)$

[5] Junkar, I., Cvelbar, U. and Lehocky, M., "Plasma treatment of biomedical materials", Mater. Tehnol., 45 (3), 221$226(2011)$

[6] Lehocký, M. and Mráček, A., "Improvement of dye adsorption on synthetic polyester fibers by low temperature plasma pre-treatment, Czech. J. Phys., 56, B1277-B1282 (2006)

[7] Tobolova, M. and Kresalek, V., "Edible oil comparison by terahertz time domain spectroscopy system Tera OSCAT", Latest Trends on Systems - Volume I, 134-137 (2014)

[8] MenloSystems, "Tera OSCAT datasheet", online, [cited 09/19/2014] available: 〈http://www.photonicsolutions.co.uk/datasheets/menlo/MENLO-OSCAT.pdf>

[9] Gavenda, T. and Křesálek, V., "Terahertz time-domain spectroscopy for distinguishing different kinds of gunpowder", Proc. of SPIE Vol. 8900, 89000H-1-89000H-6 (2013) 